\title{
Aplicación de un ciclo de innovación docente en la asignatura "Catálisis para la Industria y el Medio Ambiente" del Grado en Química
}

\section{Application of an educational innovation model in the "Industrial and Environmental Catalysis" subject given in the Chemistry Degree}

LUIS F. BOBADILLA BALADRÓN

ORCID: http://orcid.org/0000-0003-0085-9811

Universidad de Sevilla

Departamento de Química Inorgánica e-mail: lbobadilla@us.es

Fecha de recepción:

Fecha de aceptación:

DOI: http://dx.doi.org/10.12795/9788447221912.077 Pp.: 1777-1794 


\section{Resumen}

La presente comunicación incluye los resultados de la aplicación de un ciclo de mejora e innovación docente en la asignatura optativa "Catálisis para la Industria y el Medio Ambiente" impartida en el tercer curso del grado en Química. En este trabajo se abordan una serie de principios didácticos aplicados en 8 horas de sesiones teóricas en los que se propone un modelo metodológico con una serie de contenidos y unos criterios de evaluación. Este modelo pretende mejorar el grado de aprendizaje de los estudiantes e incrementar su capacidad de atención y su grado de participación.

Palabras clave: Catálisis para la Industria y el Medio Ambiente; Grado en Química; Docencia universitaria; Experimentación docente universitaria.

\section{Abstract}

This article includes the results obtained in the application of an educational innovation model for improving the quality of the optative subject entitled "Industrial and Environmental Catalysis" given in the Chemistry Degree. In this work were evaluated a series of didactical principles applied during 8 hours of some theoretical sessions in which is proposed a methodological model that includes several insides and evaluation criterial. This model claims to enhance the learning level awakening the interest of the student and overcoming the barriers to classroom participation.

Keywords: Industrial and Environmental Catalysis; Chemistry Degree; University teaching; University teaching experimentation 


\section{Descripción del contexto}

La asignatura "Catálisis para la Industria y el Medio ambiente" es una asignatura de carácter optativo que se imparte en el tercer curso del Grado en Química y el Doble Grado en Química e Ingeniería de Materiales. Yo soy el coordinador de esta asignatura e imparto toda la parte de teoría, los seminarios asociados y algunos de los grupos de la parte práctica. La asignatura consta de un total de 24 h teóricas, $6 \mathrm{~h}$ de seminarios y 15 h de prácticas de laboratorio. El ciclo de mejora se ha implementado en un periodo de 8 horas correspondientes a 8 sesiones de la parte teórica de la asignatura.

\section{Diseño del ciclo de mejora}

Con el objetivo de analizar el grado de conocimiento inicial de los estudiantes, he diseñado un cuestionario de diagnóstico inicial para los estudiantes que incluye tres pequeñas cuestiones y los alumnos deben responder el primer día que se aplica el ciclo.

\section{Propuesta del reto: problema inicial}

La producción de poliestireno es un proceso fundamental en la industria química para generar polímeros con numerosas aplicaciones (carcasas de televisores, de impresoras, juguetes, perchas, cajas de CD, cuchillas de afeitar, indumentaria deportiva, etc). En la figura 1 aparecen representadas dos rutas posibles, una ruta no sostenible (Fig.1a) y otra sostenible (Fig. 1b), para producir poliestireno a escala industrial. 


\section{LUIS F. BOBADILLA BALADRÓN}
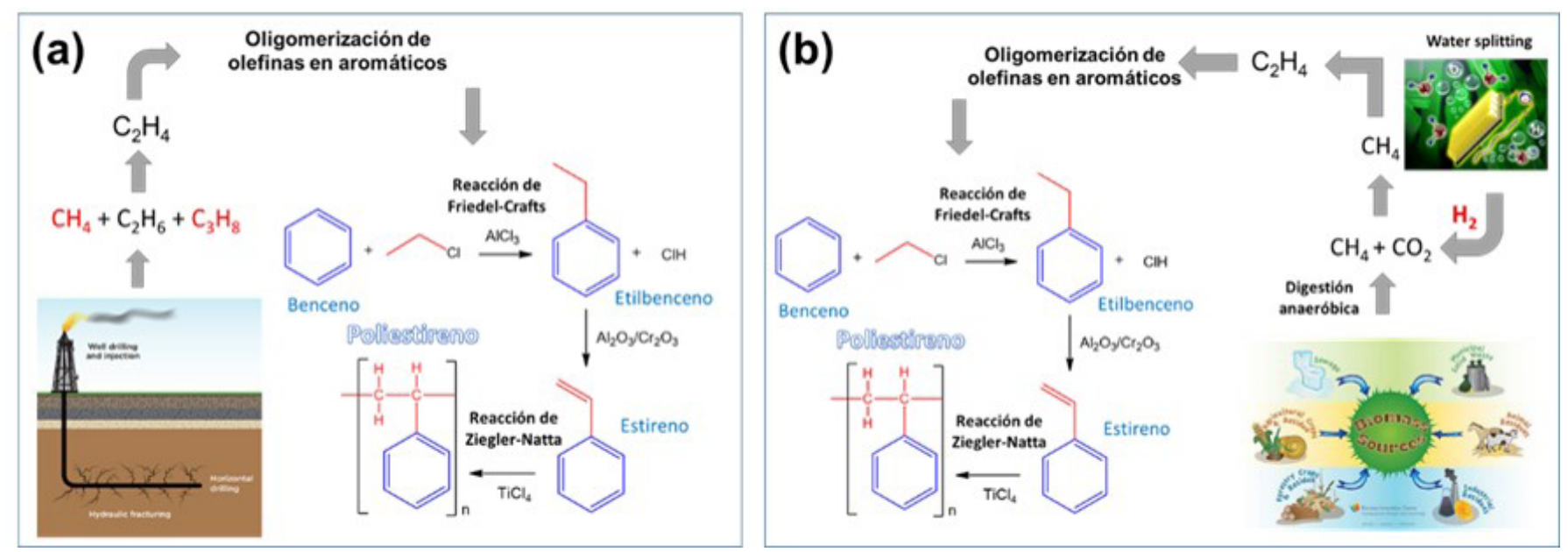

Figura 1. Posibles rutas de producción de poliestireno a escala industrial usando un proceso no sostenible (a) y otro sostenible (b)

El alumno debe responder a las siguientes preguntas de forma directa y concisa:

1) ¿Qué importancia tienen los catalizadores en ambos procesos?

2) ¿Qué diferencia existe entre un proceso sostenible y un proceso no sostenible?

3) ¿Podrían plantearse otras rutas? Sugiere alguna que se te ocurra.

Jornadas de Formación e Innovación Docente del Profesorado I № 2 (2019) 


\section{Mapa de contenidos}

En la figura 2 aparece esquematizado el mapa de contenidos con el problema planteado en el centro. El mapa de contenidos incluye una serie fundamental de contenidos conceptuales que el alumno deberá asimilar para después aplicarlos y analizar con detalle las diferentes etapas envueltas en ambos procesos químicos propuestos como retos. A través de estos conceptos, el alumno desarrollará la destreza para ser capaz de reconocer e identificar las diferentes reacciones envueltas y los catalizadores que se utilizan en cada reacción, así como adquirir habilidades que le permitan procesar toda la información relevante. Este modelo debe dar a los alumnos una nueva visión sobre las aplicaciones de la catálisis para alcanzar un desarrollo sostenible y desarrollar un pensamiento crítico sobre la viabilidad de los procesos.

Jornadas de Formación e Innovación Docente del Profesorado | № 2 (2019) Esta obra se distribuye con la licencia Creative Commons 


\section{LUIS F. BOBADILLA BALADRÓN}

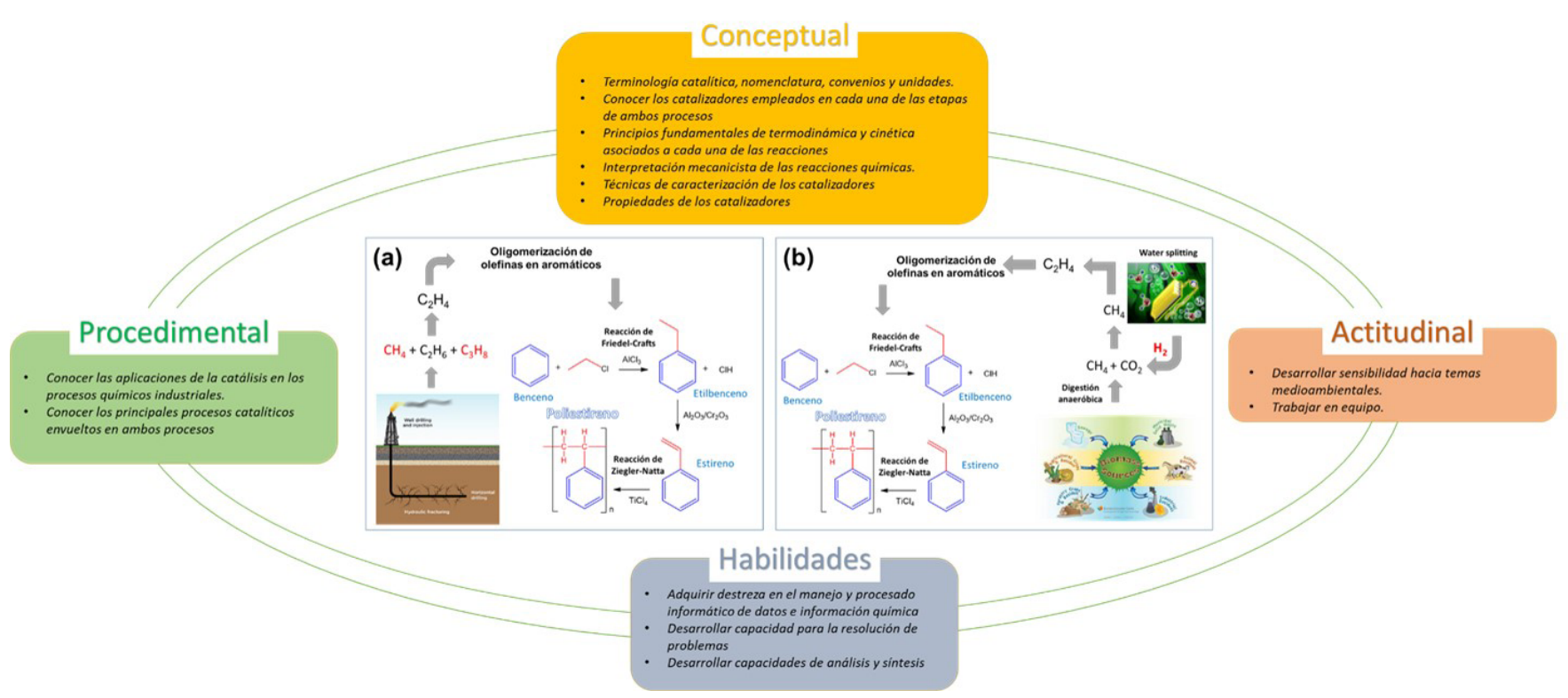

Figura 2. Mapa de contenidos incluyendo en el centro el problema planteado

Jornadas de Formación e Innovación Docente del Profesorado I № 2 (2019)

cc (7) $\Theta$ Esta obra se distribuye con la licencia Creative Commons

Internacional (CC BY-NC-ND 4.0.) 
Los dos retos propuestos aparecen representados en el centro. El objetivo final de ambos retos es producir poliestireno, aunque en el reto 1 se propone un proceso no sostenible y en el reto 2 un proceso sostenible, y los alumnos deben encontrar los diferentes pasos que forman ambos procesos analizando las diferentes etapas catalíticas y comprendiendo por qué en un caso el proceso es sostenible y el otro no. Los alumnos han formado grupos de trabajo para trabajar por separado cada una de las etapas y al final se hará una puesta en común y un debate sobre el proceso global. Es necesario que hagan una búsqueda bibliográfica y vean la importancia de cada uno de los pasos. El debate final será también importante y aprenderán como un proceso tan complejo requiere múltiples competencias y un importante trabajo en equipo.

\section{Modelo metodológico posible}

Basándome en otros ciclos de mejoras en el aula y tratando que el alumno tenga una participación mayor en las clases he planteado el modelo metodológico que aparece representado en la figura 3 . El modelo está diseñado para dos ciclos de 4 horas cada uno. Cada ciclo se inicia planteando a los alumnos una pregunta abierta o un reto relacionada con el tema que voy a explicar para que piensen sobre ella y busquen posibles respuestas fuera del aula. A continuación, hago una exposición del tema explicándoles los nuevos conceptos y mostrándoles ejemplos aplicados que les ayuden a entenderlos. Intento que los alumnos participen y trato de llamar su atención continuamente haciéndoles preguntas de estilo socrático. Al mismo tiempo que voy explicando los contenidos del tema, les voy proponiendo una serie de ejercicios y problemas para que trabajen sobre ellos fuera del aula. Una vez finalizado el tema, los alumnos salen a resolver los problemas a la pizarra y en común resolvemos las dudas que le hayan podido surgir. La última sesión de este método está dedicada a una discusión general sobre las respuestas que los 
alumnos han propuesto para resolver el reto planteado en la sesión inicial. De esta forma finaliza el ciclo y se inicia un nuevo ciclo en que se plantea un nuevo reto inicial relacionado con el siguiente tema.

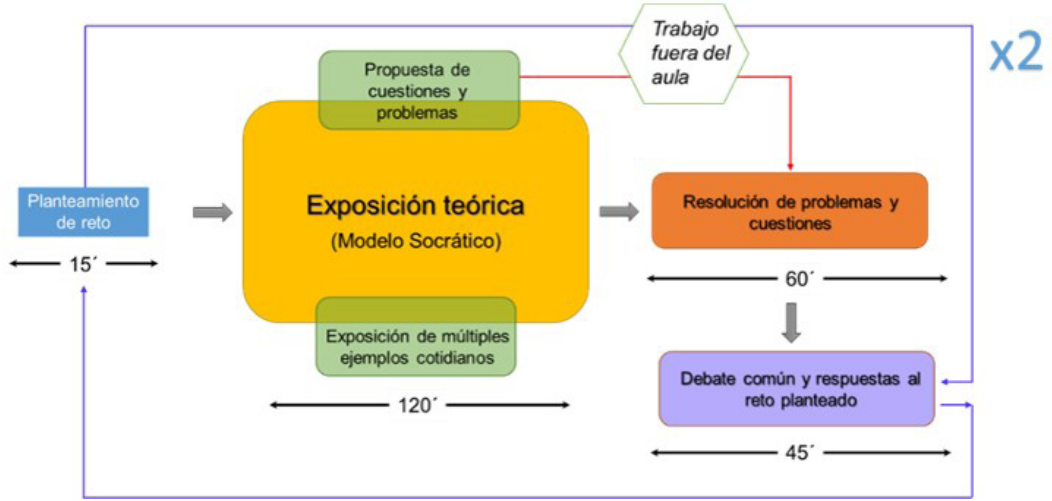

Figura 3. Modelo metodológico posible

\section{Secuencia de actividades}

Las actividades programadas en el modelo metodológico pueden dividirse en varias sesiones didácticas que han sido previamente planificadas. En total hay 8 sesiones, cuatro para cada ciclo del modelo. En la tabla 1 se detallan secuencialmente todas las actividades programadas para las 8 sesiones.

Tabla 1. Secuencia de actividades del ciclo de mejora

SESIÓN 1: Planteamiento de un reto

Duración: $15^{-}$

\section{Descripción:}

Les planteo a los alumnos un reto que consiste en la propuesta de un proceso químico que envuelve varias etapas catalíticas para producir poliestireno partiendo de una materia prima no renovable. Los alumnos deben investigar sobre cada una de las etapas y analizar si es posible desarrollar otras rutas.

RECURSOS: Intervención del profesor

Jornadas de Formación e Innovación Docente del Profesorado I № 2 (2019) Esta obra se distribuye con la licencia Creative Commons Reconocimiento-NoComercial-SinObraDerivada Internacional (CC BY-NC-ND 4.0.) 


\section{Descripción:}

En esta sesión del ciclo se imparte la parte teórica del tema mostrando los conceptos fundamentales, aunque intento que no sea una clase magistral en la que únicamente yo hable, sino que intento que los alumnos participen planteándoles preguntas continuamente y enseñándoles ejemplos muy aplicados para que vean el sentido que tiene lo que vemos. Al mismo tiempo, les voy proponiendo cuestiones y problemas para que trabajen sobre ellos fuera del aula y en la siguiente sesión resolverlos en clase.

RECURSOS: Utilización de transparencias (recursos electrónicos) y de la pizarra para dar las explicaciones

\begin{tabular}{|l|l}
\hline SESIÓN 3: Resolución de cuestiones y problemas & Duración: 60
\end{tabular}

\section{Descripción:}

Los alumnos saldrán a la pizarra a resolver los problemas que fueron propuestos en la sesión anterior. Las dudas que surjan serán resueltas en común e intentaré que mi participación en esta sesión sea considerablemente reducida.

RECURSOS: Utilización de la pizarra para resolver los problemas

SESIÓN 4: Debate sobre las respuestas al reto inicial Duración: 45

\section{Descripción:}

Se trata de una sesión de debate en la que los alumnos exponen sus ideas y sus respuestas al reto inicial. Mi papel aquí será moderar el debate y dejar que los discutan entre ellos. Finalmente tratamos de llegar a una conclusión general y a un consenso si hay diferentes opiniones.

RECURSOS: Moderador de debate

SESIÓN 5-8: Nuevo ciclo de 4 sesiones Duración: $4 \mathrm{~h}$

\section{Descripción:}

Las siguientes $4 \mathrm{~h}$ se procede de forma análoga a las cuatro primeras incluyendo un nuevo reto y explicando un nuevo tema. En este caso, el reto es análogo al primero pero el proceso es sostenible y la materia prima de partida es renovable. El objetivo final será que los alumnos comparen ambos procesos y debatan sobre el concepto de sostenibilidad. 


\section{Aplicación del ciclo de mejora docente}

\section{Relato de mi experiencia personal durante las sesiones}

Este es mi tercer año como docente, y la implantación del CIMA me ha supuesto un reto personal que me ha hecho salir de mi zona de confort a la hora de impartir la docencia. Hasta ahora, mi modelo habitual estaba basado en clases en las que el principal protagonista era yo y con el nuevo modelo he tenido que abordar el reto de darle un mayor protagonismo a los estudiantes sin saber cómo iban a responder.

En la primera de las sesiones les expliqué el reto al que se iban a enfrentar y les planteé el problema inicial. A continuación, les pasé el cuestionario inicial y tuvieron un tiempo aproximado de 10 minutos para responderlo. Como el problema propuesto tenía que ser trabajado en grupos de 3-4 alumnos, el resto del tiempo de la sesión fue dedicado a formar los diferentes grupos y a definir los objetivos que debería trabajar cada uno.

Las siguientes cinco sesiones fueron desarrolladas siguiendo el plan preestablecido en la secuencia de actividades y combinando clases explicativas mediante exposición de los temas y resolviendo problemas sobre los que alumnos previamente trabajaban tanto en clase como fuera del aula. En todo momento, he intentado hacer a los alumnos protagonistas lanteando cuestiones de forma continuada y los alumnos han estado bastante participativos. 
Las últimas dos sesiones han estado dedicadas a exponer la resolución del problema planteado en la primera sesión, saliendo cada uno de los grupos a la pizarra para presentar sus resultados. Todos los alumnos han participado y han estado bastante activos mediante el proceso grupal de resolución del problema. La última sesión terminó entregando nuevamente a los alumnos el cuestionario de diagnóstico inicial para que volvieran a responderlo y poder evaluar las escalaras de aprendizaje.

¿Qué ha funcionado durante el desarrollo de las sesiones? Me ha sorprendido el grado de participación y de responsabilidad de los alumnos. Han demostrado que saben trabajar en equipo y el trabajo que han hecho ha estado muy compensado. Los alumnos se han mostrado resolutivos y han sido muy protagonistas a lo largo de las sesiones. Con respecto a lo que no ha funcionado, hay que decir también que hay aspectos que no han ido como yo esperaba. Me hubiese gustado un debate más activo entre todos y no que cada uno expusiese sus resultados sin que hubiese ningún compañero que se las rebatiese. Por otro lado, también ha pesado mi falta de experiencia a la hora de establecer el planteamiento del problema inicial, ya que el problema era muy extenso y podía abordarse desde múltiples perspectivas. La experiencia de los alumnos les impide analizar con detalle todas ellas y esto ha podido ser un hándicap a la hora de generar un debate más abierto entre los diferentes grupos. Por tanto, encontrar un planteamiento de problema adecuado es fundamental para mejorar la implantación de mi ciclo de mejora. Finalmente, la gestión del tiempo es otro de los aspectos que debo mejorar para tratar de adecuar mejor el tiempo dedicado a las exposiciones de los alumnos y el tiempo que yo dedico a mis explicaciones. En conclusión, puedo decir que la experiencia ha sido positiva, pero hay algunos aspectos que deben ser mejorados para aumentar la calidad de la docencia impartida. 


\section{Evaluación del aprendizaje de los alumnos: escaleras de aprendizaje}

A través de los cuestionarios de evaluación realizados antes y después de aplicar el ciclo de mejora docente se puede obtener el progreso experimentado en los alumnos. Las respuestas del cuestionario final se contrastan con las del cuestionario inicial categorizándolas en grupos o categorías de respuestas similares (escalones). Cada una de las categorías supone un nivel de aprendizaje que va de menor a mayor y sus límites marcan las dificultades (Porlán, R. 2017). Las siguientes figuras presentan las escaleras de aprendizaje asociadas a los cuestionarios inicial y final planteadas a los alumnos.

Pregunta 1. ¿Qué importancia tienen los catalizadores en ambos procesos?

En la primera pregunta (Figura 4), inicialmente puede observarse que hay un importante porcentaje de alumnos que da la respuesta más simple sin mencionar ninguno de los aspectos que definen a un catalizador en sí. Hay otro porcentaje que menciona alguno de los parámetros que caracterizan a un catalizador. Sin embargo, no hay ninguno que de una definición completa. En el cuestionario final se observa una importante mejoría en la que aparece un $17 \%$ de los alumnos que dan una definición bastante completa del concepto de catalizador. Hay otro grupo intermedio que se mantiene y sólo menciona algunos de los parámetros. En este grupo, el número total de alumnos es aproximadamente el mismo en el cuestionario inicial y final, aunque se produce una inversión entre los que respondieron la respuesta $\mathrm{B}$ y la respuesta C. Por otro lado, es significativa la reducción del número de alumnos que ya no da una definición simple del proceso. 
Cuestionario inicial

$0 \%$

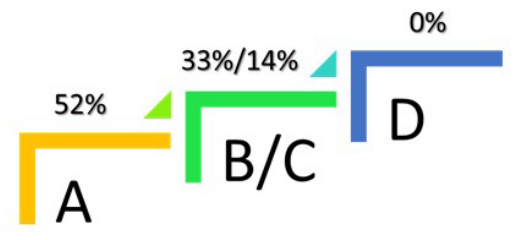

Cuestionario final

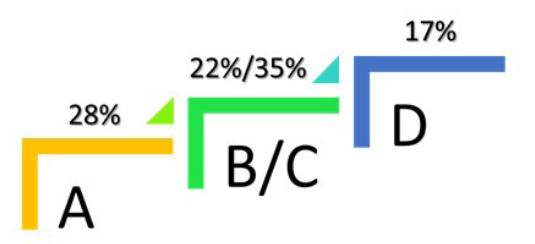

A: Su presencia es necesaria para que ocurra el proceso

B: Permiten que ocurra el proceso y disminuyen la energía de activación

C: Permiten que ocurra el proceso y aumentan la selectividad

D: Permiten que ocurra en proceso, disminuyen la energía de activación y aumentan la selectividad

Figura 4. Escaleras de aprendizaje asociadas a la primera cuestión

Pregunta 2. ¿Qué diferencia existe entre un modelo sostenible y uno no sostenible?

En la figura 5 aparecen representadas las escaleras de aprendizaje para la segunda cuestión. En esta pregunta se ha observado una mejoría muy significativa en el aprendizaje. Hay un pequeño grupo de alumnos que dan una respuesta simple y que se mantiene en número. Sin embargo, hay un porcentaje mayoritario que inicialmente daba las respuestas C y D, y en el cuestionario final se reduce notablemente y pasan a dar las respuestas de mayor complejidad E y $F$.

Es muy importante que los alumnos hayan subido un peldaño en esta escalera de aprendizaje, ya que la pregunta propuesta pretende que los alumnos debatan sobre un tema fundamental para esta asignatura. El concepto de desarrollo sostenible es un tema muy actual, pero cuando se pide a un alumno que discuta sobre este tema con una visión científica más profunda suelen aparecer algunas limitaciones que como futuros científicos deben superar. 
Cuestionario inicial

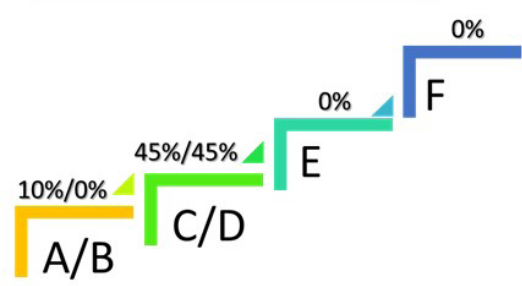

\section{Cuestionario final}

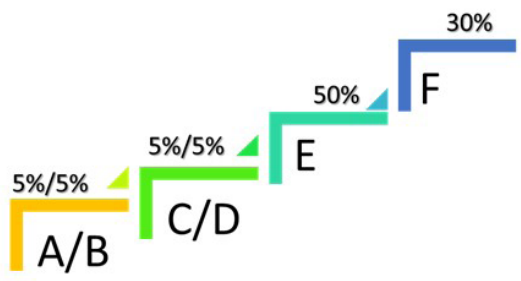

A: Un proceso no sostenible genera un impacto medioambiental

B: Un proceso sostenible es más económico

C: En un proceso sostenible la materia prima es renovable

D: Un proceso no sostenible genera residuos contaminantes

E: Un proceso sostenible usa materias primas renovables y no genera residuos

F: Un proceso sostenible usa materias primas renovables y no genera residuos. Aporta ejemplos ilustrativos

Figura 5. Escaleras de aprendizaje asociadas a la segunda cuestión

Pregunta 3. ¿Podrían plantearse otras rutas? Sugiere alguna que se te ocurra

Como puede observarse en la figura 6 , en la tercera cuestión es donde menos cambios se han observado. Esta cuestión engloba un mayor nivel de complejidad y el alumno debe aportar unos conocimientos que no tiene por qué haber adquirido previamente. A pesar de ello, es notable el porcentaje de alumnos que da alguna respuesta más compleja a pesar de la dificultad de la cuestión. Puede observarse también una pequeña anomalía, y es que inicialmente había un $10 \%$ que daba la respuesta más compleja y al final no hay ninguno. Este hecho es debido a que tras hacer la actividad, los alumnos han centrado más su respuesta en $C$ y han preferido no arriesgarse en $\mathrm{D}$ proponiendo rutas alternativas. 
Cuestionario inicial

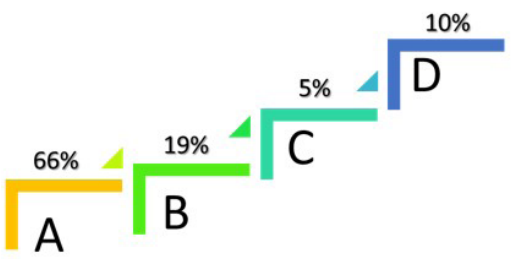

Cuestionario final

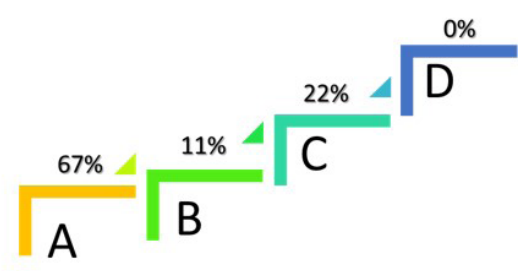
A: No responde nada
B: Responde Si o No
C: Son posibles otras rutas con otros catalizadores o reactivos
D: Existen otras rutas con otros catalizadores o reactivos. Propone alguna alternativa

Figura 6. Escaleras de aprendizaje asociadas a la tercera cuestión

Puede concluirse entonces que en la primera cuestión los alumnos muestran una mejoría discreta, en la segunda una mejoría notable y en la tercera no hay ninguna mejoría debido a la dificultad de la cuestión.

\section{Evaluación del ciclo de mejora docente}

\section{Aspectos a mantener y cambios para un futuro ciclo de mejora}

Uno de las cosas que mejor ha funcionado en el modelo de mejora aplicado ha sido la organización y la participación de los alumnos en grupos de trabajo. Los alumnos han mostrado una actitud dinámica y han pasado a ser el centro de atención en el aula. Por otro lado, las cuestiones del diagnóstico inicial me han permitido analizar el modelo mental que presentaban inicialmente los alumnos y la comparación con el diagnóstico final ha servido para conocer la evolución de estos modelos preestablecidos. Sin embargo, hay algunos aspectos del modelo que deben ser mejorados. Por ejemplo, el problema reto planteado 
ha sido demasiado complejo como demuestra la tercera escalera de aprendizaje. Una de las tareas en las que trabajaré si sigo aplicando este modelo de mejora será la búsqueda de problemas reto más adecuados y optimizados para el alumno.

\section{Incorporación a la práctica docente habitual}

A través de esta experiencia, hay varios aspectos que incluiré en mi práctica docente habitual: (i) construcción de escaleras de aprendizaje a partir de cuestionarios iniciales y finales, (ii) centrar más la atención en los alumnos y dinamizar más las aulas usando como modelo el libro de Finkel (Finkel, D. 2008), (iii) desarrollo de mapas de contenidos coherentes y planificar secuencias de actividades bien organizadas en sesiones, y (iv) escribir un diario de clase más profundo para analizar con más detalle mi experiencia docente y construir sobre él mi modelo didáctico personal.

\section{Principios didácticos que han guiado mi experiencia}

De forma personal, este curso ha ejercido una influencia muy positiva y me ha aportado una nueva visión para impartir mi docencia. Como docente universitario, creo que es algo fundamental que nos orienten y nos "enseñen a enseñar" (Bain, K. 2007). Para transmitirles los contenidos de la asignatura a los alumnos es necesario establecer un vínculo con ellos y conocer sus modelos mentales. A partir de aquí se deben organizar los contenidos que se van a enseñar y se debe preparar un modelo metodológico para impartirlos. El establecimiento de mapas conceptuales y la relación organizada de actividades debe hacerse antes de aplicar el modelo. Para que el modelo funcione es necesario llevar a cabo una organización y tratar de seguir el 
modelo una vez elaborado, tratando de improvisar lo mínimo posible. Sólo así se puede conseguir el objetivo establecido inicialmente. Por último, la evaluación del modelo es básica para conocer la evolución del aprendizaje de los alumnos y autoevaluarnos a nosotros mismos para ver si conseguimos que los alumnos cambien sus modelos mentales y adquieran una nueva forma de pensar sobre los contenidos. Las escaleras de aprendizaje me parecen una herramienta muy útil para la evaluación y me han permitido visualizar de forma directa como ha funcionado mi modelo. El diario personal nos ayuda a comprender los aspectos que debemos mejorar en nuestro modelo y que cosas han funcionado mejor o peor. A partir de aquí, espero aplicar todos estos conceptos en mis futuras tareas docentes. 


\section{Referencias bibliográficas}

Porlán, R. (2017). Enseñanza universitaria. Cómo mejorarla. Madrid: Ediciones Morata, S.L.

Finkel, D. (2008). Dar clase con la boca cerrada. Valencia: Publicaciones Universidad de Valencia.

Bain, K. (2007). Lo que hacen los mejores profesores de univerșidad. Valencia: Publicaciones Universidad de Valencia. 